\title{
Video Article \\ Retinal Detachment Model in Rodents by Subretinal Injection of Sodium Hyaluronate
}

\author{
Hidetaka Matsumoto ${ }^{1}$, Joan W. Miller ${ }^{1}$, Demetrios G. Vavvas ${ }^{1}$ \\ ${ }^{1}$ Retina Service, Angiogenesis Laboratory, Department of Ophthalmology, Massachusetts Eye and Ear Infirmary, Harvard Medical School \\ Correspondence to: Hidetaka Matsumoto at hide-m@gunma-u.ac.jp, Demetrios G. Vavvas at Demetrios_Vavvas@meei.harvard.edu
}

URL: https://www.jove.com/video/50660

DOI: doi:10.3791/50660

Keywords: Medicine, Issue 79, Photoreceptor Cells, Rodentia, Retinal Degeneration, Retinal Detachment, animal models, Neuroscience, ophthalmology, retina, mouse, photoreceptor cell death, retinopathy, age-related macular degeneration (AMD)

Date Published: 9/11/2013

Citation: Matsumoto, H., Miller, J.W., Vavvas, D.G. Retinal Detachment Model in Rodents by Subretinal Injection of Sodium Hyaluronate. J. Vis. Exp. (79), e50660, doi:10.3791/50660 (2013).

\section{Abstract}

Subretinal injection of sodium hyaluronate is a widely accepted method of inducing retinal detachment (RD). However, the height and duration of RD or the occurrence of subretinal hemorrhage can affect photoreceptor cell death in the detached retina. Hence, it is advantageous to create reproducible RDs without subretinal hemorrhage for evaluating photoreceptor cell death. We modified a previously reported method to create bullous and persistent RDs in a reproducible location with rare occurrence of subretinal hemorrhage. The critical step of this modified method is the creation of a self-sealing scleral incision, which can prevent leakage of sodium hyaluronate after injection into the subretinal space. To make the self-sealing scleral incision, a scleral tunnel is created, followed by scleral penetration into the choroid with a $30 \mathrm{G}$ needle. Although choroidal hemorrhage may occur during this step, astriction with a surgical spear reduces the rate of choroidal hemorrhage. This method allows a more reproducible and reliable model of photoreceptor death in diseases that involve RD such as rhegmatogenous RD, retinopathy of prematurity, diabetic retinopathy, central serous chorioretinopathy, and age-related macular degeneration (AMD).

\section{Video Link}

The video component of this article can be found at https://www.jove.com/video/50660/

\section{Introduction}

Photoreceptor cell death and subsequent visual decline occurs when the photoreceptors are separated from the underlying retinal pigment epithelium. Physical separation of photoreceptors is seen in various retinal disorders, including age-related macular degeneration (AMD), central serous chorioretinopathy, diabetic retinopathy, and retinopathy of prematurity, as well as rhegmatogenous (i.e. caused by a break in the retina) retinal detachment (RD). Subretinal injection of sodium hyaluronate is a widely accepted model to create an RD that leads to photoreceptor cell death, providing insight into the pathophysiology of photoreceptor degeneration ${ }^{1-15}$.

Photoreceptor degeneration induced by subretinal sodium hyaluronate injection, first introduced in $2001^{7}$, has the advantage of a reasonable time course (days to weeks). However, it can have significant variability of photoreceptor cell loss from animal to animal due to two major factors that affect photoreceptor cell death after RD: 1) height and duration of the RD, and 2) the occurrence of subretinal hemorrhage. There is a steep technical learning curve to the method that contributes to both factors. Photoreceptor cell degeneration increases with the height of the RD, as the distance between the retinal pigment epithelial (RPE) layer and photoreceptors increases ${ }^{16-17}$. Consistent with these reports, our previous experiments showed more photoreceptor death in bullous RDs than shallow RDs. It has also been reported that subretinal hemorrhage is toxic for photoreceptor cells and affects photoreceptor cell death ${ }^{18-21}$. Likewise, we have observed more photoreceptor death in RDs with subretinal hemorrhage than RDs without subretinal hemorrhage. Hence, techniques to minimize variability have to focus on achieving consistent heights of $\mathrm{RD}$ while avoiding subretinal hemorrhage.

Our modified method of inducing RD can make reproducible bullous and persistent RD at the same position of the eye with rare occurrence of subretinal hemorrhage. We performed surgery using a temporal approach because it is easier to achieve a wider operative field compared with other sites. After the conjunctival incision, a self-sealing scleral incision is made using a $30 \mathrm{G}$ needle. A scleral tunnel is created, followed by scleral penetration into the choroid. If choroidal bleeding occurs in this step, the hemorrhage will exit the eye through the scleral wound, and the bleeding can be stopped by astriction with a surgical spear. An anterior chamber puncture is then performed from the cornea to reduce intraocular pressure. This is an important step because subretinal injection alone will lead to increased intraocular pressure with resultant retinal artery occlusion and ischemia of the inner retina. A $33 \mathrm{G}$ needle connected to a Hamilton $10 \mu \mathrm{l}$ syringe is then inserted into the subretinal space, and $3.5 \mu \mathrm{l}$ sodium hyaluronate is gently injected to detach the neurosensory retina from the underlying RPE. In contrast to other methods of inducing RD that are performed under fundus observation, this technique is performed under direct observation. Since the scleral wound is self-sealing, sodium hyaluronate will not leak out after injection. Finally, glue is put on the scleral wound, and the conjunctiva is reattached to its original position. These lasts steps also reduce the risk of sodium hyaluronate leakage. The injection of $3.5 \mu$ sodium hyaluronate creates reproducible RDs ( $50 \%$ of the retina) in the eyes of 8 -week-old mice. Creating the self-sealing wound is the most important step in our modified 
procedure because it prevents the injected sodium hyaluronate from leaking out of the eye, thus allowing reproducible bullous and persistent RDs.

Protocol

All experiments on animals were performed in accordance with the Association for Research in Vision and Ophthalmology Statement for the Use of Animals in Ophthalmic and Vision Research, and the guidelines and regulations set forth by the Massachusetts Eye and Ear Infirmary Animal Care Committee.

\section{Pretreatment}

1. Anesthetize an 8-week-old mouse with an intraperitoneal injection of a mixture of $60 \mathrm{mg} / \mathrm{kg}$ ketamine and $6 \mathrm{mg} / \mathrm{kg}$ xylazine.

2. Cut the whiskers. This is done because they interfere with visualization and instrument placement.

3. Dilate the pupil with $5 \%$ phenylephrine and $0.5 \%$ tropicamide.

4. Cut the cilia. This is also done because they interfere with visualization and instrument placement.

5. Apply topical anesthesia ( $0.5 \%$ proparacaine hydrochloride ophthalmic solution).

\section{Surgery Under the Microscope}

1. Set the mouse in a lateral position with the nose toward the surgeon. A toe pinch is performed to confirm surgical anesthesia, and sterile gloves are donned prior to starting surgery.

2. Incise the temporal conjunctiva at the posterior limbus and separate the conjunctiva from the sclera. Avoid penetrating the sclera. After this step, grasp the conjunctiva at the limbus with forceps to control the eye.

3. Make a self-sealing scleral incision using the tip of a $30 \mathrm{G}$ needle with the bevel pointed up. Make a scleral tunnel through the sclera and penetrate the sclera into the choroid (Figure 1). Avoid penetrating the retina. If choroidal bleeding occurs and comes out through the scleral wound, perform an astriction with a surgical spear until the bleeding stops. This scleral wound will be self-sealed with intraocular pressure.

4. Puncture the cornea with a $30 \mathrm{G}$ needle to reduce intraocular pressure. Use the needle parallel to the iris to avoid injuring the iris and lens during entry into the anterior chamber. The intraocular pressure will cause the wound to self-seal.

5. Insert a $33 \mathrm{G}$ needle connected to a Hamilton $10 \mu \mathrm{l}$ syringe into the subretinal space with the bevel pointed down (towards the inner eye), and inject $3.5 \mu \mathrm{l}$ sodium hyaluronate gently to detach the neurosensory retina from the underlying RPE. Avoid penetrating the retina with the 33 $\mathrm{G}$ needle because sodium hyaluronate will go into the vitreous space but not the subretinal space if the retina is penetrated. Also, avoid rapid injection because it will create an oral tear, and sodium hyaluronate will leak into the anterior chamber.

6. Let the aqueous humor flow out from the corneal puncture by pushing the cornea around the corneal puncture with forceps to adjust the intraocular pressure.

7. Confirm absence of leakage from the scleral wound using a surgical spear.

8. To reduce the risk of sodium hyaluronate leakage, place cyanoacrylate surgical glue on the scleral wound.

9. Reattach the conjunctiva to its original position using cyanoacrylate surgical glue. This will further reduce the risk of sodium hyaluronate leakage.

10. Check the fundus using a cover glass and confirm the creation of a bullous RD without subretinal hemorrhage.

\section{After Treatment}

1. Apply bacitracin antibiotic ointment to the eye to reduce the risk of infection.

2. Keep the mice on a heating pad to prevent the anesthesia (mixture of ketamine and xylazine) from causing low body temperature subsequent to low blood pressure.

3. Put the mice back to the animal cage after they awaken from anesthesia. Monitor the mice daily for complications. If any complications arise, euthanize the mice as described below.

\section{Sacrifice}

1. Euthanize the mice by cervical dislocation after intraperitoneal injection of $100 \mathrm{mg} / \mathrm{kg}$ sodium pentobarbital, and enucleate the eyes at appropriate time points for each experiment (not shown). Avoid applying pressure on the eye during enucleation because sodium hyaluronate may leak out. Bullous RDs should be persistent for at least 14 days.

\section{Representative Results}

To assess the persistence of the RD made by this protocol, cryosections were made on days 3, 7, and 14 after induction of RD. Six eyes were used for each time point. Hematoxylin and eosin (HE) staining was used to visualize the sections. All sections showed a bullous RD approaching the lens (Figure 2). One eye showed subretinal hemorrhage on day 7 . No eyes showed any signs of infection or lens injury. 


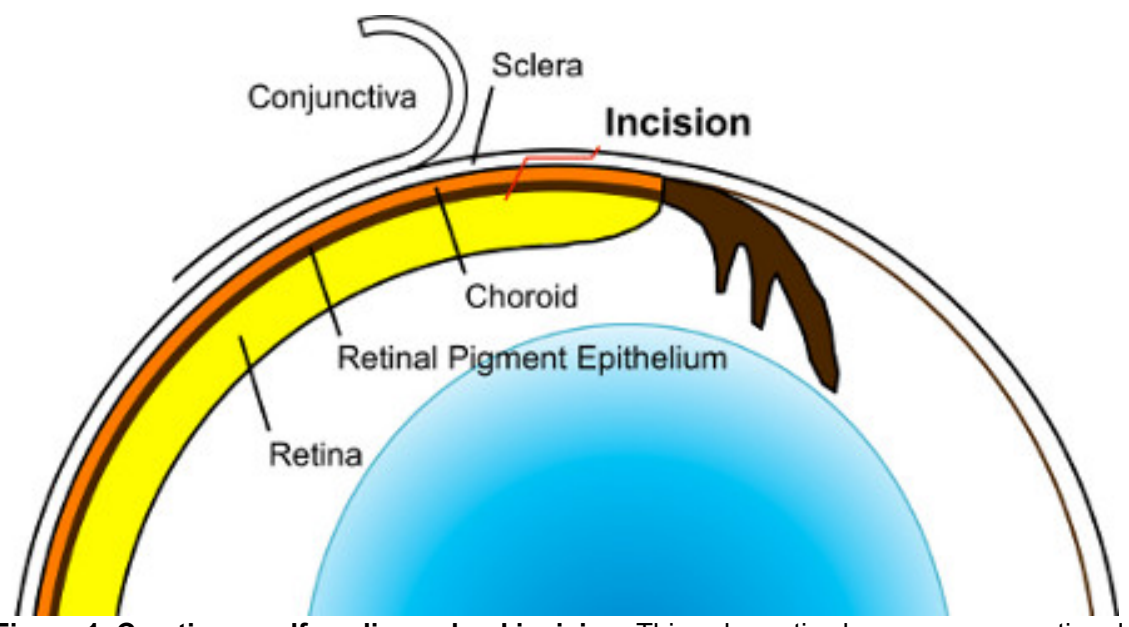

Figure 1. Creating a self-sealing scleral incision. This schematic shows a cross-sectional image of a normal mouse eye. Red line indicates the self-sealing scleral incision. A scleral tunnel followed by scleral penetration into the choroid with a $30 \mathrm{G}$ needle creates a self-sealing scleral incision. This scleral wound will be self-sealed with intraocular pressure, which can prevent leakage of sodium hyaluronate after injection into the subretinal space and achieve persistent bullous retinal detachments.
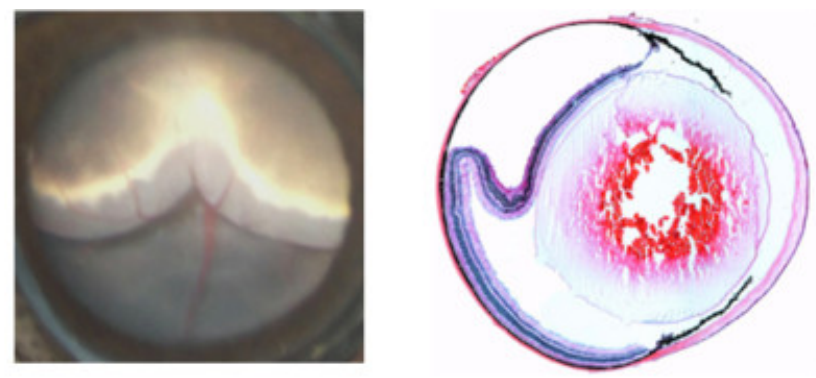

Do

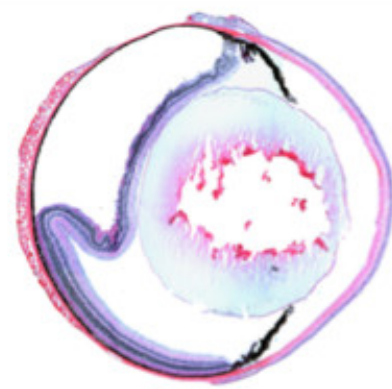

D3

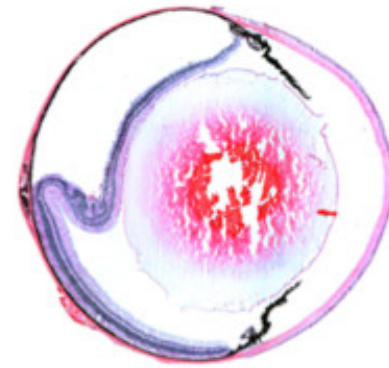

D7

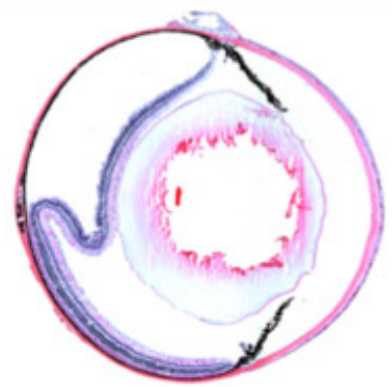

D14

Figure 2. Time course of retinal detachment. This fundus photo shows a bullous retinal detachment without subretinal hemorrhage immediately after surgery. Cryosections with hematoxylin and eosin (HE) staining show persistent bullous retinal detachment without subretinal hemorrhage through at least day 14.

\section{Discussion}

Several methods for establishing an RD model in rodent eyes have been reported ${ }^{3-15,22}$. Most of them utilize subretinal injection of sodium hyaluronate because it is a viscous material commonly used during intraocular surgery in humans, and it is not associated with any known ocular toxicity ${ }^{1-15}$. Sodium hyaluronate, rather than normal saline or phosphate-buffered saline (PBS), increases the duration of the RD.

The methods for subretinal injection of sodium hyaluronate use one of two approaches: a transvitreal approach ${ }^{3-6}$ or a transscleral approach $^{7-15}$ Both methods are performed with observation of the fundus. In the transvitreal approach, a subretinal injector is introduced into the vitreous cavity, a retinotomy is created in the peripheral retina, and the sodium hyaluronate is injected into the subretinal space. In this method, two retinal tears are made, which increases the risk of retinal hemorrhage that might go into the subretinal space. In addition, there is a risk of lens injury when the retinotomy is created. There are several modified methods for the transscleral approach. In most of these methods ${ }^{7-12}$, after reducing intraocular pressure with an anterior chamber puncture, a $30 \mathrm{G}$ needle connected to a syringe filled with sodium hyaluronate is directly inserted into the subretinal space through the conjunctiva, sclera, choroid, and RPE. The sodium hyaluronate is then injected into the subretinal space. The risk of retinal tear and lens injury using this transscleral method is less than that using the transvitreal approach. However, the hole made in the sclera by a $30 \mathrm{G}$ needle is large, especially for mouse eyes, and the sodium hyaluronate injected into the subretinal space easily leaks out of 
the eye through the scleral wound. This leads to a lower, less persistent RD and more variable photoreceptor cell death. Moreover, if choroidal bleeding occurs in the scleral perforation step, the hemorrhage will spread into the subretinal space because the intraocular pressure has been reduced prior to the subretinal hyaluronate injection.

Several factors can influence the effect of RD on the detached retina, including both subretinal hemorrhage and the height and persistence of $\mathrm{RD}^{16-21}$. Photoreceptor cell death increases with increasing height of $\mathrm{RD}^{16,17}$, and photoreceptor cell damage may be more extensive because of reduced diffusion of oxygen and essential nutrients from the choriocapillaris with higher RD compared to shallow RD. Subretinal hemorrhage is also toxic for photoreceptor cells ${ }^{18-21}$; possible mechanisms of this toxicity in the detached retina include hypoxia and metabolic disruption by subretinal hemorrhage as a diffusion barrier, and direct neurotoxicity induced by blood components (such as iron). Lens injury, which has been reported to have a protective effect on retinal ganglion cells ${ }^{23}$, may also affect photoreceptor cell death after induction of RD. In addition, if the entry wound site is not sealed, sodium hyaluronate may leak out with eye manipulation during enucleation. This may lead to erroneous classification of an RD as shallow, which may in turn affect the interpretation of the results.

We modified the transscleral method for subretinal injection of sodium hyaluronate to increase the reproducibility of RDs and reduce the rate of subretinal hemorrhage. The critical step of this protocol is creating a self-sealing scleral incision using a $30 \mathrm{G}$ needle, which prevents leakage of sodium hyaluronate after injection. Unlike previous methods, this protocol is performed without observation of the fundus so greater attention is paid to the scleral wound. Application of glue also prevents the sodium hyaluronate from leaking out of the eye. In our experience, the rate of subretinal hemorrhage with this protocol was significantly less than that with other protocols. If choroidal bleeding occurs during the scleral incision step, it will exit the eye through the scleral wound because this step is performed before reducing intraocular pressure. If choroidal bleeding does occur after reduction of the intraocular pressure and detachment of the neurosensory retina, blood will dissect into the subretinal space. We found this to happen in about $5 \%$ of the cases in contrast to about $10-20 \%$ with the other techniques. These animals should be excluded from analysis.

This technique can also be used for subretinal injection of vector-mediated gene transfer to target photoreceptor or RPE cells ${ }^{24,25}$. Because the typical vehicle (PBS, saline) for these injections is significantly less viscous than sodium hyaluronate, standard techniques are plagued by more leaks. The technique described herein, by reducing this risk, makes vector transfer experiments more reproducible and reliable.

\section{Disclosures}

The Massachusetts Eye and Ear Infirmary has an ownership interest in three U.S. patents directed to the use of verteporfin. In addition, the Massachusetts Eye and Ear Infirmary has ownership interest in certain patent applications directed to the selective destruction of subretinal choroidal neovasculature for the treatment of macular degeneration and other disorders. The Massachusetts Eye and Ear Infirmary receives royalties as a result of these patents and patent applications and Dr. Miller receives a share of the same in accordance with the Massachusetts Eye and Ear Infirmary's institutional Patent Policy and Procedures, which includes royalty-sharing provisions. The author, Dr. Joan W. Miller, is a past board member and former shareholder of Alcon, Inc., and currently serves as a consultant for Alcon; Imagen Biotech, Inc.; ISIS Pharmaceuticals; Kalvista Pharmaceuticals; Regeneron Pharmaceuticals, Inc and ONL Therapeutics, LLC. Dr. Demetrios G. Vavvas serves as consultant for Roche, Genentech, Kala Pharmaceuticals.

\section{Acknowledgments}

We thank Wendy Chao for her support in critical review. This work was supported by Bausch \& Lomb Vitreoretinal Fellowship (HM), National Eye Institute grant EY014104 (JWM), Research to Prevent Blindness Foundation (DGV), Lions Eye Research Fund (DGV), and a generous donation of the Yeatts family (JWM and DGV).

\section{References}

1. Anderson, D.H., Guerin, C.J., Erickson, P.A., Stern, W.H., \& Fisher, S.K. Morphological recovery in the reattached retina. Invest. Ophthalmol. Vis. Sci. 27, 168-183 (1986).

2. Cook, B., Lewis, G.P., Fisher, S.K., \& Adler, R. Apoptotic photoreceptor degeneration in experimental retinal detachment. Invest. Ophthalmol. Vis. Sci. 36, 990-996 (1995).

3. Zacks, D.N., et al. Caspase Activation in an Experimental Model of Retinal Detachment. Invest. Ophthalmol. Vis. Sci. 44, 1262-1267, doi:10.1167/iovs.02-0492 (2003).

4. Zacks, D.N., Han, Y., Zeng, Y., \& Swaroop, A. Activation of Signaling Pathways and Stress-Response Genes in an Experimental Model of Retinal Detachment. Invest. Ophthalmol. Vis. Sci. 47, 1691-1695, doi:10.1167/iovs.05-1209 (2006).

5. Nakazawa, T., et al. Characterization of cytokine responses to retinal detachment in rats. Mol. Vis. 12, 867-878, doi:v12/a98 [pii] (2006).

6. Nakazawa, T., et al. Tumor Necrosis Factor-Mediates Photoreceptor Death in a Rodent Model of Retinal Detachment. Invest. Ophthalmol. Vis. Sci. 52, 1384-1391, doi:10.1167/iovs.10-6509 (2011).

7. Hisatomi, T., et al. Relocalization of apoptosis-inducing factor in photoreceptor apoptosis induced by retinal detachment in vivo. Am. J. Pathol. 158, 1271-1278, doi:S0002-9440(10)64078-3 [pii] 10.1016/S0002-9440(10)64078-3 (2001).

8. Hisatomi, T., et al. Critical role of photoreceptor apoptosis in functional damage after retinal detachment. Curr. Eye Res. 24, 161-172 (2002).

9. Nakazawa, T., et al. Monocyte chemoattractant protein 1 mediates retinal detachment-induced photoreceptor apoptosis. Proc. Natl. Acad. Sci. 104, 2425-2430, doi:10.1073/pnas.0608167104 (2007).

10. Trichonas, G., et al. Receptor interacting protein kinases mediate retinal detachment-induced photoreceptor necrosis and compensate for inhibition of apoptosis. Proc. Natl. Acad. Sci. 107, 21695-21700, doi:10.1073/pnas.1009179107 (2010).

11. Roh, M.I., Murakami, Y., Thanos, A., Vavvas, D.G., \& Miller, J.W. Edaravone, an ROS Scavenger, Ameliorates Photoreceptor Cell Death after Experimental Retinal Detachment. Invest. Ophthalmol. Vis. Sci. 52, 3825-3831, doi:10.1167/iovs.10-6797 (2011). 
12. Mantopoulos, D., et al. Tauroursodeoxycholic acid (TUDCA) protects photoreceptors from cell death after experimental retinal detachment. PLoS One. 6, e24245, doi:10.1371/journal.pone.0024245 PONE-D-11-03511 [pii] (2011).

13. Yang, L., Bula, D., Arroyo, J.G., \& Chen, D.F. Preventing retinal detachment-associated photoreceptor cell loss in Bax-deficient mice. Invest. Ophthalmol. Vis. Sci. 45, 648-654, doi: 10.1167/iovs.03-0827 (2004).

14. Cebulla, C.M., Ruggeri, M., Murray, T.G., Feuer, W.J., \& Hernandez, E. Spectral domain optical coherence tomography in a murine retinal detachment model. Exp. Eye Res. 90, 521-527, doi:10.1016/j.exer.2010.01.008 (2010).

15. Secondi, R., Kong, J., Blonska, A.M., Staurenghi, G., \& Sparrow, J.R. Fundus Autofluorescence Findings in a Mouse Model of Retinal Detachment. Invest. Ophthalmol. Vis. Sci. 53, 5190-5197, doi:10.1167/iovs.12-9672 (2012).

16. Machemer, R. Experimental retinal detachment in the owl monkey. IV. The reattached retina. Am. J. Ophthalmol. 66, 1075-1091 (1968).

17. Ross, W., Lavina, A., Russell, M., \& Maberley, D. The correlation between height of macular detachment and visual outcome in macula-off retinal detachments of < or = 7 days' duration. Ophthalmology. 112, 1213-1217, doi:S0161-6420(05)00283-6 [pii] 10.1016/ j.ophtha.2005.01.040 (2005).

18. Glatt, H. \& Machemer, R. Experimental subretinal hemorrhage in rabbits. Am. J. Ophthalmol. 94, 762-773 (1982).

19. Toth, C.A., Morse, L.S., Hjelmeland, L.M. \& Landers, M.B. 3rd. Fibrin directs early retinal damage after experimental subretinal hemorrhage. Arch. Ophthalmol. 109, 723-729 (1991).

20. Benner, J.D., Hay, A., Landers, M.B. 3rd, Hjelmeland, L.M., \& Morse, L.S. Fibrinolytic-assisted removal of experimental subretinal hemorrhage within 7 days reduces outer retinal degeneration. Ophthalmology. 101, 672-681 (1994).

21. Bhisitkul, R.B., et al. Neuroprotective effect of intravitreal triamcinolone acetonide against photoreceptor apoptosis in a rabbit model of subretinal hemorrhage. Invest. Ophthalmol. Vis. Sci. 49, 4071-4077, doi: 10.1167/iovs.08-1892 (2008).

22. Zeng, R., Zhang, Y., Shi, F. \& Kong, F. A Novel Experimental Mouse Model of Retinal Detachment: Complete Functional and Histologic Recovery of the Retina. Invest. Ophthalmol. Vis. Sci. 53, 1685-1695, doi:10.1167/iovs.11-8241 (2012).

23. Leon, S., Yin, Y., Nguyen, J., Irwin, N., \& Benowitz, L.I. Lens injury stimulates axon regeneration in the mature rat optic nerve. J. Neurosci. 20, 4615-4626 (2000).

24. Murakami, Y., et al. Inhibition of choroidal neovascularization via brief subretinal exposure to a newly developed lentiviral vector pseudotyped with Sendai viral envelope proteins. Hum. Gene Ther. 21, 199-209, doi:10.1089/hum.2009.102 (2010).

25. Kong, F., et al. Self-complementary AAV5 vector facilitates quicker transgene expression in photoreceptor and retinal pigment epithelial cells of normal mouse. Exp. Eye Res. 90, 546-554, doi:10.1016/j.exer.2010.01.011 (2010). 